\title{
Profile of Tuberculosis in Paediatric patients
}

\author{
Sandesh KS ${ }^{1}$, Praveenkumar Sindhur ${ }^{2}$, Pooja Shah ${ }^{3}$, Anish Singhal ${ }^{4}$ \\ ${ }^{1}$ Dr. Sandesh KS, Department of PG Studies in Social Work, Nehru Memorial College, Sullia, DK, Karnataka, $\quad{ }^{2}$ Dr. \\ Praveenkumar Sindhur, Department of Pediatrics, KVG Medical College Sullia, DK, Karnataka, ${ }^{3}$ Dr. Pooja Shah, \\ Department of Microbiology, SBHGMC Dhule, Maharashtra, ${ }^{4}$ Dr. Anish Singhal, Department of Physiology, KVG \\ Medical College, Sullia, DK, Karnataka, India.
}

Address for Correspondence: Dr. Sandesh KS, Email: drsandesh2@gmail.com

\begin{abstract}
Introduction: Tuberculosis (TB) is very important cause of infection related deaths worldwide and has been declared by $\mathrm{WHO}$ as a global health emergency. Around half million children fall ill with TB each year. Due to non-specific signs and symptoms TB in children may be overlooked. Aims and objectives: 1) To study the occurrence of TB in pediatric patients at tertiary care hospital KVG Medical College, Sullia, Karnataka. 2) To study the occurrence of TB in patients with and without BCG scar. 3) To study the occurrence of smear positive cases in pediatric age group. 4) To study the type of TB in pediatric patients. Material and methods: Specimens like sputum, bronchial lavage, lymph node aspirate, gastric lavage, CSF were collected depending on the site of disease. Specimens were stained by ZN staining method. Culture was done on LJ media. Tuberculin test was also done. Chest $\mathrm{X}$ rays of the patients were done presenting with pulmonary signs and symptoms. Result: In our study, $102(0.98 \%)$ were positive. Age group of 5-9 years was the most commonly affected. Males $(57.84 \%)$ were more affected than females $(42.16 \%)$. BCG scar was found only in $20.59 \%$ cases. Smear positivity was seen in $22.89 \%$ cases. Extrapulmonary manifestations were more compared to pulmonary manifestations. Conclusion: As smear examination is negative in most of the patients, culture of Mycobacterium tuberculosis is important. This age group present with non-specific signs and symptoms and hence tuberculosis should always be considered as one of the differential diagnosis.
\end{abstract}

\section{Introduction}

India holds major tuberculosis population globally. It accounts for $20 \%$ of the newly diagnosed 8.6 million TB cases annually [1]. According to WHO estimates sputum microscopy smear-positive TB in $<14$ years accounts for $0.6 \%-3.6 \%$ of all reported cases [2]. According to some studies, pediatric tuberculosis consists of total $8-20 \%$ deaths $[3,4]$. Localized forms of illness, e.g., intrathoracic lymphadenopathy, and localized CNS disease have been reported to occur more in children [5].

Children can't cough out sputum properly and so other samples like gastric lavage and broncho-alveolar lavage has to be collected which is other major problem. In children $<5$ years specially, both diagnosis as well as treatment of tuberculosis is challenging. This is major problem where diagnostic facilities are limited. HIV and TB coinfection is difficult to treat and has high mortality and morbidity [6].

Manuscript received: $9^{\text {th }}$ January 2017

Reviewed: $16^{\text {th }}$ January 2017

Author Corrected: $24^{\text {nd }}$ January 2017

Accepted for Publication: $30^{\text {th }}$ January 2017
According to literatures, BCG vaccination helps in reducing both meningeal and disseminated tuberculosis [7].

\section{Methodology}

After obtaining the Institutional Ethics Committee approval this Prospective study was conducted for a period of 3 years from $1^{\text {st }}$ January 2014 to $31^{\text {st }}$ December 2016 at KVG Medical college, Sullia, Karnataka. Study was performed only with informed consent from the patients/legal guardian or assent from the child aged over 7 years.

Patients aged $<14$ years coming with signs and symptoms of Tuberculosis i.e cough $>2$ weeks and/or no weight gain or loss (History of unexplained weight loss or no weight gain in past 3 months; loss of weight defined as loss of more than 5\% body weight as compared to highest weight recorded in last 3 months) and/or history of contact with infected tuberculosis case were included in the study. Sputum samples were taken from the patients 
presenting with cough. If patient can't expectorate cough, gastric lavage was taken. In case of enlarged lymph nodes, lymph node aspirates were taken. In patients presenting with meningeal signs and symptoms, CSF was taken. All samples were subjected to $\mathrm{ZN}$ staining and culture was done on LJ media.

Chest $\mathrm{X}$ rays were done in patients presenting with pulmonary signs and symptoms. Tuberculin skin test using Purified protein derivative 1TU was done in all the suspected cases. Those having indurations $>10 \mathrm{~mm}$ were considered as positive.

The protocol was as follows-Sputum smear examination was taken as the primary investigation of choice. If sputum smear was positive, patient was diagnosed as smear positive pulmonary $\mathrm{TB}$ and was initiated on TB treatment. If sputum smear came negative or not available, then the patient was prescribed a course of antibiotics for duration of seven days.

In case symptoms persist, chest X-ray and Tuberculin skin test (TST) was done. In sick looking patients with persistent symptoms of $>2$ weeks duration, chest $X$ ray and TST test was performed immediately. If both chest $\mathrm{X}$ ray and TST was positive, gastric lavage or bronchoalveolar lavage was taken and if it comes to be positive it was taken as smear positive pulmonary TB and if negative smear negative pulmonary TB. If both chest Xray and TST were negative, alternate diagnosis was considered.

If in a suspected patient who has persistant symptoms and / or non-specific radiological shadows but were smear negative following 3 possible situations were considered-

1. If both TST and X-ray findings were negative, then TB is highly unlikely and an alternative diagnosis was looked for.

2. In situations where the CXR has persistence of nonspecific shadows despite a course of antibiotics, alternative samples for TB (GA/IS/BAL) were sent to establish bacteriological diagnosis irrespective of the TST positivity. In case the alternative sample was AFB positive, it was taken as smear positive case. In case these samples were negative, then an alternative diagnosis was looked for. If no alternative diagnosis was established, the case was classified and treated as smear negative TB.

3. If only TST was positive and X-ray chest was not suggestive, then an extra- pulmonary site or an alternative diagnosis was suspected.

\section{Results}

A total 10358 cases were suspected of Tuberculosis and amongst them 102 were positive.

Table-1: Prevalence of tuberculosis.

\begin{tabular}{|c|c|}
\hline Total suspected & 10358 \\
\hline Total positive & 102 \\
\hline Occurrence & $0.98 \%$ \\
\hline
\end{tabular}

Shows the occurrence of TB to be $0.98 \%$

Table-2: Age and Sex distribution in patients having TB.

\begin{tabular}{|c|c|c|c|}
\hline Age & Males & Females & Total \\
\hline Birth-4 years & $17(16.67 \%)$ & $12(11.76 \%)$ & $29(28.43 \%)$ \\
\hline 5years-9years & $24(23.53 \%)$ & $17(16.67 \%)$ & $41(40.20 \%)$ \\
\hline 10years-14 years & $18(17.65 \%)$ & $14(13.73 \%)$ & $32(31.37 \%)$ \\
\hline Total & $\mathbf{5 9}(\mathbf{5 7 . 8 4} \%)$ & $\mathbf{4 3 ( 4 2 . 1 6 \% )}$ & $\mathbf{1 0 2}(\mathbf{1 0 0} \%)$ \\
\hline
\end{tabular}

Amongst 102 positive cases, $57.84 \%$ were males and $42,16 \%$ were females. The male: female ratio was found to be $1.37: 1$.

Amongst 102 positive cases, sputum could be obtained in 83 cases. 
Table-3: Smear positives as done by ZN (Ziel and Nelson) staining

\begin{tabular}{|c|c|}
\hline Total smear positive & $19(22.89 \%)$ \\
\hline Total smear negative & $64(77.11 \%)$ \\
\hline Total sputum samples & 83 \\
\hline
\end{tabular}

The sputum by ZN staining was positive in 19 cases (18.63\% of total cases).

Culture positive cases are shown in table 4

Table-4: TB patients having culture positive on Lowenstein Jenson media

\begin{tabular}{|c|c|}
\hline Total culture positive & $69(67.65 \%)$ \\
\hline Total culture negative & $33(32.35 \%)$ \\
\hline Total samples tested for culture & 102 \\
\hline
\end{tabular}

Culture positivity was found in $67.65 \%$ of cases.

Tuberculin test positive cases are showin in Table 5.

Table-5: TB patients having tuberculin test positive

\begin{tabular}{|c|c|}
\hline Total tuberculin positive & $69(83.13 \%)$ \\
\hline Total tuberculin negative & $14(16.87 \%)$ \\
\hline Total cases in which tuberculin test was done & 83 \\
\hline
\end{tabular}

Tuberculin test could be done in 83 cases and of which 69 (83.13\%) cases were positive.

Type of tuberculosis prevalent in children-

Table-6: Type of tuberculosis prevalent in pediatric age group.

\begin{tabular}{|c|c|}
\hline Type of TB & Percentage \\
\hline Pulmonary & $39(38.24 \%)$ \\
\hline Lymph node & $35(34.31 \%)$ \\
\hline Meningeal & $17(16.67 \%)$ \\
\hline Disseminated & $11(10.78 \%)$ \\
\hline
\end{tabular}

This result shows that Lungs and Lymph Nodes were the most common sites of TB in our patients.

Difference between occurrence in cases with BCG scars and without BCG scar-

Table 7: Occurrence of tuberculosis in patients with BCG scar and without BCG scar

\begin{tabular}{|c|c|}
\hline Patients having TB and have BCG scar & $21(20.59 \%)$ \\
\hline Patients having TB and without BCG scar & $81(79.41 \%)$ \\
\hline Total & $102(100 \%)$ \\
\hline
\end{tabular}

Data shows that $20.59 \%$ of TB cases had a BCG scar.

\section{Discussion}

About $40 \%$ of the Indian population is estimated to be infected with TB bacteria, majority of which have latent TB [8]. In our study, out of 10358 clinically suspected tuberculosis cases, 102 were positive. So, the occurrence of tuberculosis in present study was $0.98 \%$
(Table 1). According to Global tuberculosis control, the prevalence of TB in pediatric population is $0.6-3.6 \%$ [9]. Till 2012 TB burden in children was unknown, when it was first estimated to be 0.5 million cases (around 5\%) and 64000 deaths among children [10]. As per 2012 
survey, globally around 2 million deaths occurred due to $\mathrm{TB}[10]$. Of the remaining 10256 , most of our patients got benefitted either by antibiotic therapy or by treatment for some other differential diagnosis. According to statistics the prevalence of TB 2013 in Indian population was $2.6 \%[11]$.

In our study, 5-9 years age group was most affected age group and overall $52 \%$ cases were males (Table:2). Suryanarayana et al showed that majority of cases $(34.80 \%)$ were in $5-9$ years age group and $51 \%$ of males were affected compared to $49 \%$ females. which correlates well with our study [12].

We support their observation that such results might be due to the fact that this age group children are mostly school going with exploratory behaviour; and males are exposed more to the outside environment compared to females.

Since the bacilli enters the sputum making the patient infectious to others sputum smear testing is done. But for definitive diagnosis of tuberculosis, positive culture of a diagnostic specimen is very important [13].

The most frequent sample used from a patient with a persistent and productive cough is sputum. In our study, $22.89 \%$ cases were smear positive and $67.65 \%$ were culture positive (Table: 3 and 4).

Childhood TB diagnosis is a major challenge, as bacteriologic confirmation is hardly achieved as sputum smear microscopy is often the only diagnostic test available. Positive findings with probable TB are found for around $<10 \%-15 \%$ of cases [14]. According to a Chinese study in 1990 the smear positivity was 30\% $[15,16]$ Farjana Rahman et al in their study showed a culture positivity of $68.7 \%$.[17] All the above findings correlates with our study results.

In our study tuberculin test was positive in $83.13 \%$ of cases (table no:5), which is similar to Fernandiz et al finding of $81 \%$ tuberculin test positivity [18]. The tuberculin most widely used is purified protein derivative (PPD), which is derived from cultures of M. tuberculosis. In our study pulmonary TB was found in $38.24 \%$ cases (table no 6) and extrapulmonary manifestations were found in $62.76 \%$ cases.

Amongst extra pulmonary, lymph node tuberculosis was most common. This correlates with the study of Saumya Swaminathan et al (32-43\%) [4]. Extrapulmonary TB was $46 \%$ in the study of Sanjay Jain et al (46\%) [19]. More cases of meningeal and disseminated TB in our study may be due to the fact that most of the children were without BCG scar and BCG protects against pulmonary and disseminated TB.

In our study, BCG scar was found in $20.59 \%$ cases. According to Kabra et al, more prevalence of TB was there in non immunized patients [20].

\section{Conclusion}

As smear examination is negative in most of the patients, culture of Mycobacterium tuberculosis is important. This age group present with non specific signs and symptoms and hence tuberculosis should always be considered as one of the differential diagnosis.

Funding: Nil, Conflict of interest: None initiated, Perission from IRB: Yes

\section{References}

1. Lienard J, Carlsson F. Murine Mycobacterium marinum Infection as a Model for Tuberculosis. Methods Mol Biol. 2017;1535:301-315.

2. John TJ, Vashishtha VM, John SM. 50 years of tuberculosis control in India: progress, pitfalls and the way forward. Indian Pediatr. 2013 Jan 8;50(1):93-8.

3. Kabra SK, Lodha R, Seth V. Tuberculosis in children-what has changed in last 20 years? Indian J Pediatr. 2002 Nov;69 Suppl 1:S5-10.

4. S.Swaminathan and B. Rekha,"Pediatric tuberculosis: global overview and challenges," Clinical Infectious Diseases, 2010; 50(3):S184-S194.

5. Marais BJ, Gie RP, Schaaf HS, Hesseling AC, Enarson DA, Beyers N. The spectrum of disease in children treated for tuberculosis in a highly endemic area. Int $\mathrm{J}$ Tuberc Lung Dis. 2006 Jul;10(7):732-8.

6. Deepak Arora, Neerja Jindal, Renu Bansal3, Shilpa Arora. Rapid Detection of Mycobacterium tuberculosis in Sputum Samples by Cepheid Xpert Assay: A Clinical Study. Journal of Clinical and Diagnostic Research. 2015 May; 9(5): DC03-DC05.

7. Paniker C K. Anantnarayan \& Paniker's Textbook of Microbiology. Chapter 35 Haemophilus. $8^{\text {th }}$ edn. Universities press India Private Limited. Chennai. 2009. 329-34. 
8. TB Statistics for India - National \& state statistics. http:// www. tbfacts. org/tb-statistics-india/\#sthash.d6 DMVlcu.dpuf.

9. Global tuberculosis control-epidemiology, strategy, financing. WHO Report 2009. http://www.who. int/tb /publications/global_report/2009/ en/index.html. 31 August 2009.

10. Lienard J, Carlsson F. Murine Mycobacterium marinum Infection as a Model for Tuberculosis. Methods Mol Biol. 2017;1535:301-315.

11. TB India 2015. Annual Status Report 2015. http:// www. tbcindia.nic.in/showfile.php?lid=3166

12. L. Suryanarayana, H.V. Suryanarayana and P.S. Jagannatha. Prevalence of Pulmonary tuberculosis among children in a south Indian Community. Ind. J. Tub $1999 ; 46: 171-8$

13. Knechel NA. Tuberculosis: pathophysiology, clinical features, and diagnosis. Crit Care Nurse. 2009 Apr;29(2):34-43; quiz 44. doi: 10.4037/ccn2009968.

14. Marais BJ, Graham SM, Cotton MF, Beyers N. Diagnostic and management challenges for childhood tuberculosis in the era of HIV. J Infect Dis. 2007 Aug 15;196 Suppl 1:S76-85.
15. Cruz AT, Starke JR. Clinical manifestations of tuberculosis in children. Paediatr Respir Rev. 2007 Jun; 8 (2):107-17. Epub 2007 Jun 5.

16. Eamranond P, Jaramillo E. Tuberculosis in children: reassessing the need for improved diagnosis in global control strategies. Int J Tuberc Lung Dis. 2001 Jul;5 (7):594-603 .

17. Farjana Rahman, Saurab Kishore Munshi, S. M. Mostofa Kamal, A. S. M. Matiur Rahman, M. Majibur Rahman, and Rashed Noor. Comparison of Different Microscopic Methods with Conventional TB Culture. Stamford Journal of Microbiology. 2011 Oct; 1(1): 4650 .

18. Fernández de Larrea C, Fandiño C, López D, del Nogal B, Rodríguez N, Convit J, Araujo Z, de Waard JH. [Tuberculosis in subjects under 15 years of age in the population of Warao in Venezuela]. Invest Clin. 2002 Mar;43(1):35-48.

19. Sanjay K. Jain, Alvaro Ordonez, Aarti Kinikar, et al. Pediatric Tuberculosis in Young Children in India: A Prospective Study. BioMed Research International, vol. 2013, Article ID 783698, 7 pages, 2013. doi:10. $1155 / 2013 / 783698$.

20. Kabra SK, Lodha R, Seth V. Some current concepts on childhood tuberculosis. Indian J Med Res. 2004 Oct; 120(4):387-97.

\section{How to cite this article?}

Sandesh KS, Praveenkumar Sindhur, Pooja Shah, Anish Singhal. Profile of Tuberculosis in Paediatric patients. J PediatrRes.2017;4(01):58-62.doi:10.17511/ijpr.2017.i01.12 